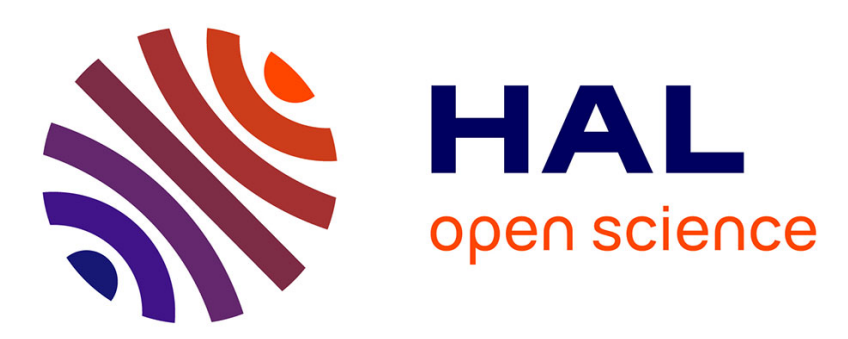

\title{
Stress concentration and instabilities in the atomistic process of brittle failure initiation
}

\author{
Sabri Souguir, Laurent Brochard, Karam Sab
}

\section{To cite this version:}

Sabri Souguir, Laurent Brochard, Karam Sab. Stress concentration and instabilities in the atomistic process of brittle failure initiation. International Journal of Fracture, 2020, 224 (2), pp.235-249. 10.1007/s10704-020-00459-x . hal-03170412

\section{HAL Id: hal-03170412 \\ https://hal.science/hal-03170412}

Submitted on 16 Mar 2021

HAL is a multi-disciplinary open access archive for the deposit and dissemination of scientific research documents, whether they are published or not. The documents may come from teaching and research institutions in France or abroad, or from public or private research centers.
L'archive ouverte pluridisciplinaire HAL, est destinée au dépôt et à la diffusion de documents scientifiques de niveau recherche, publiés ou non, émanant des établissements d'enseignement et de recherche français ou étrangers, des laboratoires publics ou privés. 


\title{
Stress concentration and instabilities in the atomistic process of brittle failure initiation
}

\author{
Sabri Souguir - Laurent Brochard* - Karam Sab
}

Received: date / Accepted: date

\begin{abstract}
A major challenge in the macroscopic modeling of brittle failure initiation is to reconcile stressdriven failure in absence of stress concentration and energy-driven failure under high stress concentration (crack). In this paper, we perform athermal molecular simulations to investigate the underlying physics behind stress- to energy-driven failures. In the athermal limit, the evolution of an atomic system is deterministic and is obtained by energy minimization. Failure is expected when the system suddenly bifurcates to a broken configuration which can be formally evaluated as an atomic instability characterized by a negative eigenvalue of the Hessian matrix. We applied this methodology to a 2D toy model and to pristine graphene. Both stress- and energy-driven failures are triggered by an instability at the atomic scale, but the two types of failure differ widely regarding the mechanisms of instability (eigenvectors) and their multiplicity (degeneracy). With respect to existing macroscopic theories of failure initiation, these results raise some issues. In particular,
\end{abstract}

\section{S. Souguir}

Laboratoire Navier, UMR 8205, École des Ponts, IFSTTAR, CNRS, UPE

6 \& 8 avenue Blaise Pascal, 77455 Marne-la-Vallée Champssur-Marne, France.

* L. Brochard (corresponding author)

Laboratoire Navier, UMR 8205, École des Ponts, IFSTTAR, CNRS, UPE

6 \& 8 avenue Blaise Pascal, 77455 Marne-la-Vallée Champssur-Marne, France.

Tel.: +33164153788

E-mail: laurent.brochard@enpc.fr

K. Sab

Laboratoire Navier, UMR 8205, École des Ponts, IFSTTAR, CNRS, UPE

$6 \& 8$ avenue Blaise Pascal, 77455 Marne-la-Vallée Champssur-Marne, France. one should distinguish the initiation mechanisms and the physical cracking occurring after initiation, and the spatial extent of the initiation mechanism should depend on stress concentration with a minimum extent given by the ratio between toughness and strength. From an atomic scale perspective, a strain-based stability formulation seems the most appropriate. Finally, we show that the degeneracy of the modes of failure explains the size-scaling of strength and toughness at finite temperature.

Keywords brittle failure · crack initiation · strength . toughness $\cdot$ graphene

PACS 62.20.-x

\section{Introduction}

The mechanical failure of brittle materials is well understood in two limit cases: the failure in absence of stress concentration characterized by the strength $\sigma_{c}$ (criterion on stress), and the failure of cracked bodies (high stress concentration) characterized by the toughness $K_{c}$ (criterion on energy release rate). However, the failure in intermediate situations with moderate stress concentrations, are still debated in the scientific community. A typical example is the case of a sharp $\mathrm{V}$ notch, which generates a stress singularity (infinite stresses at the tip) but the exponent of the singularity is lower than $1 / 2$ so that the incremental energy release rate is 0 (Williams, 1952). In other words, irrespective of the loading, a notched material should always fail according to a stress criterion, and never fail according to an energy criterion (Leguillon, 2002). More generally, for any smooth flaw, the stress field is no more singular (Rice, 1968) so that the incremental energy release rate always falls to 0 and the energy 
criterion is not applicable. Conversely, any sharp flaw generates a stress singularity (Williams, 1952) and the stress criterion is not applicable. To address this contradiction, many theories of brittle failure initiation have been proposed in the literature which can predict failure for any stress concentration, while being consistent with the two limit cases (stress criterion at small stress concentrations, energy criterion at large stress concentrations). Let us mention the Cohesive Zone Models (Dugdale, 1960), the non local theories (Novozhilov, 1969), the Finite Fracture Mechanics (FFM) (Leguillon, 2002), and the Phase Field approaches (Miehe et al, 2010). Although these theories are all consistent with the two limit cases, their predictions differ at moderate stress concentrations, and no clear scientific consensus has emerged so far. In this respect, investigating the elementary physics at the origin of failure initiation should point to an appropriate macroscopic theory of brittle failure. All the theories were elaborated from a macroscopic point of view without detailed description of the irreversible processes occurring at the point of failure. A primary requirement is to ensure a transition from energy driven failure to stress driven failure when the size of the process zone approaches a characteristic size of the structure. Therefore, investigating the physics behind failure requires to zoom at the scale of the process zone. For cracked bodies, the scale of the process zone is given by the characteristic length $l_{c}=\left(\frac{K_{c}}{\sigma_{c}}\right)^{2}$ (ratio between toughness and the strength). $l_{c}$ ranges from a few centimeters to a nanometer depending on the material (see Fig. 1). Nanometric process zones correspond to extremely brittle materials (e.g., diamond, mono-crystals of minerals, silica glass etc.) and are small enough to be investigated with molecular simulation techniques, that is starting from the fundamental interactions between atoms. Therefore, extremely brittle materials are good candidates for a systematic study of the elementary physics behind failure initiation.

In the literature, molecular simulation has been used by many to investigate fracture propagation in brittle materials. Early works date back to the 1970's and focused primarily on simple lattice models (Thomson et al, 1971; Hsieh and Thomson, 1973; Thomson, 1986). These works highlighted some peculiarities of cracking in discrete atomic systems. In particular, crack advance is identified as a succession of snap-back instabilities corresponding to bond breaking, which paves the way to a systematic investigation of cracking at the atomic scale by looking for instabilities. Tracking instability makes it possible to investigate more complex cracking than straight propagation in a lattice model. For instance, Kitamura et al (2004) investigate eigenmodes

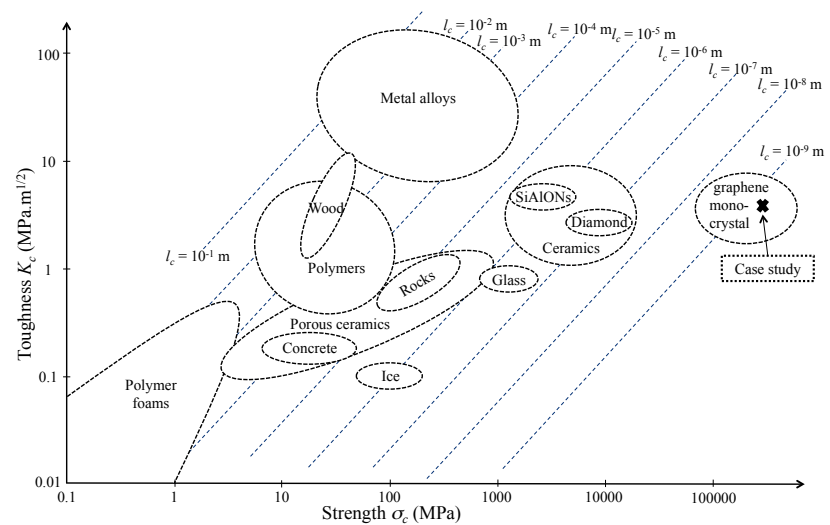

Fig. 1 Plot of strength vs toughness (adapted from Ashby (2005)). Transition between energy- and stress-driven failure requires to study the materials at the scale of the characteristic length $l_{c}=\left(\frac{K_{c}}{\sigma_{c}}\right)^{2}$. Only very brittle materials ( $l_{c}$ less than a few nanometers) can be studied by molecular simulation. Mono-crystal of pristine graphene is considered in this paper.

of failure and point the importance of small changes in crystal structures, and Li (2013) perform a similar analysis for the study of crack kinking. Delph et al (2009) show that a reasonable prediction of mode I cracking can be achieved by limiting the stability analysis to a subvolume, which significanlty improves the computational cost. Interestingly, failure initiation at moderate stress concentrations has attracted much less attention. In a previous work (Brochard et al, 2016), we investigated failure initiation under moderate stress concentrations in a 2D model of graphene. We could estimate the failure initiation behavior and confront it to macroscopic theories. In some configurations, material strength was overcome, which is not expected by many initiation theories except FFM. A specificity of FFM theory is that failure initiation is predicted by a dual criterion requiring a minimum energy release and a minimum stress over a finite length (initiation length). Situations with very high stress concentrations but little stored mechanical energy can therefore exceed the strength of the material, which is the case for the $2 \mathrm{D}$ model of graphene when periodic cracks are about to merge. At the heart of FFM theory is the fundamental principle that failure initiates as the nucleation of an initial crack over a finite length. This initiation length strongly depends on stress concentration: it is as small as the characteristic length $l_{c}$ for high stress concentrations (crack tips), but as large as the entire structure for small stress concentrations. The nucleation of a crack over a finite length suggests a description of brittle failure initiation as an unstable process, irrespective of the stress concentration, which is non obvious from a continuum perspective. Indeed, following 
fracture mechanics, some particular configurations are stable at failure: any situation for which the stress intensity decreases with the crack length at constant loading is stable (common even under force control, for instance with forces applied on crack faces). Conversely, many mechanical instabilities are not associated to failure (e.g., martensitic materials). In contrast, from an atomistic perspective, associating failure initiation and instability is consistent with the early works on lattice models, for which the process zone size is typically the size of a single bond. However, the role of stress concentration on this instability, the concept of initiation length and its relation to the characteristic length $l_{c}$ are still to be explored.

Characterizing crack initiation as an instability holds at $0 \mathrm{~K}$ (athermal limit), that is when atomic systems are frozen in their static equilibrium. An additional difficulty, though, is the effect of temperature which provides thermal agitation to atoms. Dynamics becomes essential to the evolution of the atomic systems, in particular for crack propagation (Marder and Gross, 1994). In a recent study by molecular simulation at finite temperatures, we show that, for mono-crystals, strength and toughness follow analogous scaling laws in temperature and loading rate, but differ regarding the scaling with system size (Brochard et al, 2018). The scaling in temperature and loading rate (inverse of loading time) has long been identified and is known as Zhurkov's law (Zhurkov, 1984), but the effect of system size has been disregarded so far. Yet, since strength and toughness differ only for size effects, understanding the origin of size effects should be central for the difference between energy- and stress-driven failures. Zhurkov's law is obtained by describing failure at finite temperature as a thermally activated process: failure occurs when the system reaches a transition state (saddle point of the energy landscape). The difference between strength and toughness is that strength scales with the number of atoms, whereas toughness scales with the number of cracks. A natural interpretation is that stress concentration strongly reduces the number of transition states to a few configurations in which the critical degrees of freedom are the atoms in the vicinity of the crack tip. This interpretation should be confirmed by investigating the degeneracy of the modes of instability in the athermal limit.

In this paper, we use molecular simulations in the athermal limit to investigate the occurrence of instabilities at the onset of failure for two materials: a 2D toy model and a realistic description of pristine graphene. We investigate more particularly how the modes of instability and their degeneracy depend on stress concentration. Part 2 is dedicated to the description of the methods and systems studied, part 3 to the results, and part 4 to a discussion on the implications for the macroscopic modeling of failure initiation.

\section{Methods}

In this work, the molecular simulations we refer to are 'classical' simulations, i.e., based on classical physics, in contrast with 'ab-initio' simulations based on quantum physics. Accordingly, the atoms are represented as discrete particles interacting through an empirical interaction potential $(V)$ and following Newton's dynamics. The degrees of freedom are the positions $\left(\mathbf{r}_{i}\right)$ and momenta $\left(\mathbf{p}_{i}\right)$ of the particles and the energy can be split into kinetic and potential energy: $E=\sum_{i} \frac{\mathbf{p}_{i}^{2}}{2 m_{i}}+$ $V\left(\mathbf{r}_{1}, \cdots, \mathbf{r}_{N}\right)$, where the $m_{i}$ are the masses and $N$ refers to the number of particles. To investigate the occurrence of instability at the atomic level, we used athermal simulations, i.e., simulations in the limit of 0K. In this limit, a molecular system adopts a single configuration (the ground state of minimum energy $\mathbf{r}_{i}=\mathbf{r}_{i 0}$ ) while all the momenta are null $\left(\mathbf{p}_{i}=0\right)$. Accordingly, one can limit the description of the system to the positions and interaction energy only, and disregard the momenta and kinetic energy.

Instability of discrete systems in the athermal limit has been investigated by many for a variety of irreversible processes such as the stability of crystals (Milstein and Hill, 1979), the localization of shear bands in lattices (Triantafyllidis and Bardenhagen, 1993; Bardenhagen and Triantafyllidis, 1994), the martensitic transformations (Elliott et al, 2006), the plasticity of amorphous solids (Maloney and Lemaître, 2006; Dailidonis et al, 2015), or nanoindentation-induced damages (Pratapa and Suryanarayana, 2016). The systematic approach consists in identifying 'soft modes' for which one of the eigenvalues of the Hessian matrix becomes negative. Indeed, since each particles is in static equilibrium, the total force exerted on it cancels: $\mathbf{F}_{i}=-\nabla_{\mathbf{r}_{i}} V=0$, where $\nabla_{r_{i}}$ is the gradient operator with respect to the position of particle $i$. As a consequence the Taylor expansion of the energy of the system around the ground state exhibits no first order contribution:

$$
\begin{aligned}
& E\left(\mathbf{r}_{1}, \cdots, \mathbf{r}_{N}\right) \\
& \approx E_{0}+\frac{1}{2} \sum_{i, j}\left(\mathbf{r}_{i}-\mathbf{r}_{i 0}\right)^{t} \cdot \nabla_{\mathbf{r}_{i}}\left[\nabla_{\mathbf{r}_{j}}[V]_{\langle\varepsilon\rangle}\right]_{\langle\varepsilon\rangle} \cdot\left(\mathbf{r}_{j}-\mathbf{r}_{j 0}\right) \\
& =E_{0}+\frac{1}{2} \delta \mathbf{R}^{t} \cdot \mathbb{H}_{\langle\varepsilon\rangle} \cdot \delta \mathbf{R}+O\left(\delta \mathbf{R}^{t} \cdot \delta \mathbf{R}\right)
\end{aligned}
$$

where $E_{0}=E\left(\mathbf{r}_{10}, \cdots, \mathbf{r}_{N 0}\right)$ is the energy in the ground state, the subscript $t$ represents the matrix transpose, 
and '.' refers to the usual matrix multiplication. In the above equation, the derivatives hold at constant loading of the system; and, in this work, the loading is the overall strain $\langle\varepsilon\rangle$ of the periodic cell (see details of the atomic system hereafter). The second line is a matrix reformulation with $\delta \mathbf{R}$ the vector of positions relative to the ground state and $\mathbb{H}_{\langle\varepsilon\rangle}$ the Hessian matrix of the potential. Stability of the ground state requires that the Hessian matrix is definite positive. Conversely, if one of the eigenvalues of $\mathbb{H}_{\langle\varepsilon\rangle}$ is negative, the state of the system is unstable and there exists configurations of lower energy by following an evolution in the direction of the associated eigenvector. Accordingly, one can identify an instability in the athermal limit by looking for a negative eigenvalue of the Hessian matrix, and the corresponding eigenvector characterizes the collective movements of the particles triggered by this instability, which we refer to as mode of failure. In this work, we simulate various molecular systems in the athermal limit by minimization of their interaction energy. The eigenvalues of the Hessian are evaluated while the systems are subjected to incremental mechanical loadings. Simulation ends, when the first instability is detected, and the corresponding eigenvector is computed. All the simulations were performed with LAMMPS software (http://lammps.sandia.gov) (Plimpton, 1995), complemented by LAMMPS-HESSIAN package version 3 (Costa, A. B., http://bitbucket.org/numericalsolutions/lammpshessian) to compute the Hessian matrix and an in-house program for its analysis. The LAMMPS-HESSIAN package computes the Hessian by forward finite differences, which causes a small bias detrimental for the accuracy needed in this work. We fixed this issue by modifying the package to compute the Hessian by central finite differences. We also optimized the use of memory (sparse matrix). These modification appeared critical for efficiency and accuracy of the calculations in this study. The Hessian was fully eigendecomposed, in order to study the statistical distribution of eigenvalues and its evolution with loading. Note that full decomposition comes with a significant computational cost, and much improved efficiency can be achieved by limiting the decomposition to the lowest eigenvalues (Pratapa and Suryanarayana, 2016), which would be critical to study larger systems than in the present study.

Two materials are studied : a toy model and pristine graphene (see atomic structures in Fig. 2). Confronting those two materials, one can evaluate whether the physics of failure observed for simplistic systems (closest-neighbor pair interactions) holds for more complex realistic systems (many-body potential not limited to closest neighbors), which bring confidence to the physical interpretations.
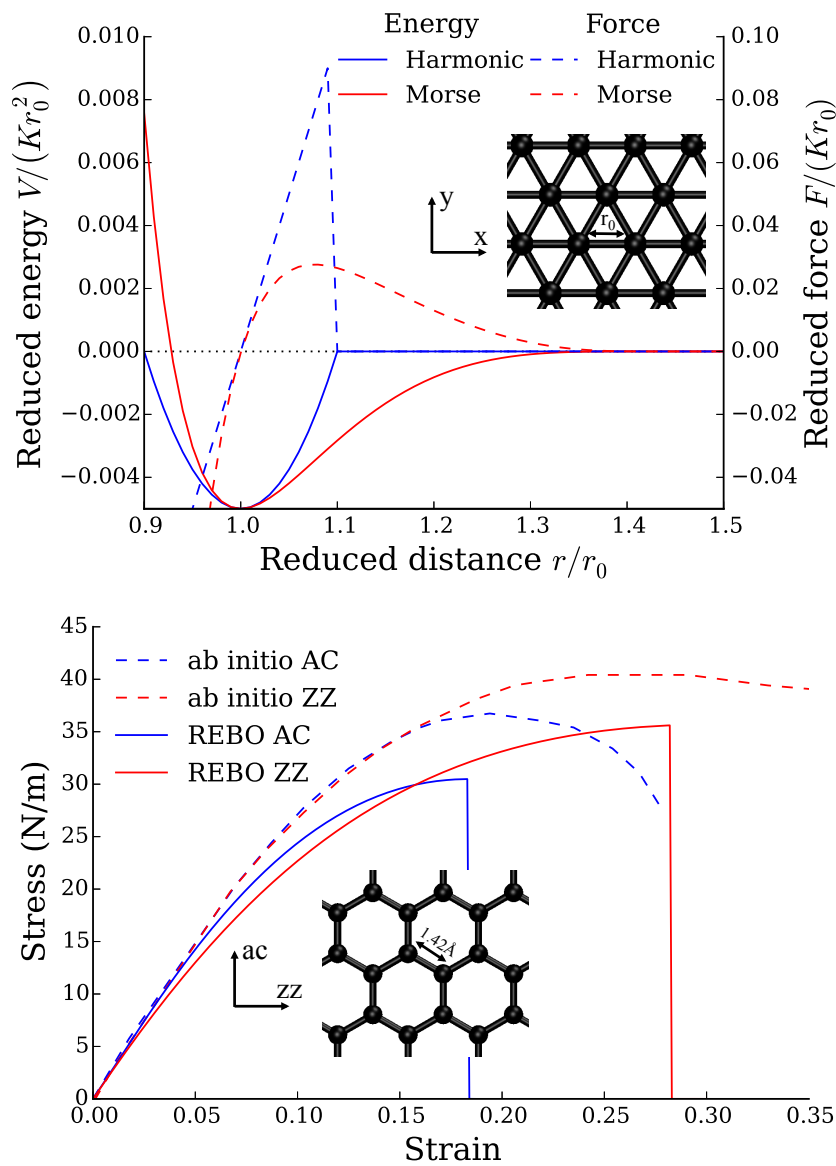

Fig. 2 The two materials studied in this work. (top) Toy model made of triangular lattice with pair interactions between closest neighbors only. A modified Morse potential is considered that ensures continuity of the potential and its derivatives. (bottom) Pristine graphene is modeled by the $\mathrm{REBO}$ reactive potential. The tensile behavior estimated with this potential compares well with ab-initio results (Liu et al, 2007).

The toy model was previously investigated in the context of the scaling laws of strength and toughness mentioned in the introduction (Brochard et al, 2018). This fictitious material has a 2D crystalline structure of regular triangular lattice and the inter-atomic interactions are limited to pair interactions between nearest neighbors. Although, it is not a real material, the simplicity of this toy model makes it possible to confront simulations and theory which proved useful for the interpretation of the scaling laws of strength and toughness. In particular, the impact of stress concentration on the scaling with size was precisely characterized for this toy model; and we intend here to relate it to the degeneracy of the mode of instability in the athermal limit. Nevertheless, the pair potential we consider in the present study differs from the harmonic potential used previously $\left(V_{H}(r)=\frac{K}{2}\left(r-r_{0}\right)^{2}\right)$. This harmonic potential was truncated at a cutoff distance 
$r_{c u t}^{H}$ to enable bond breaking and failure and shifted by $V_{\text {shift }}=\frac{K}{2}\left(r_{\text {cut }}^{H}-r_{0}\right)^{2}$ to ensure continuity of energy. But, doing so, one generates discontinuities in the derivatives of the potential at $r=r_{c u t}^{H}$ which is not suitable for a stability analysis since continuity of the second order derivatives is required. To address this issue, we considered the following modified Morse potential instead:

$$
\begin{aligned}
& V_{M}(r)= \\
& \begin{cases}D\left(1-e^{-\alpha\left(r^{2}-r_{0}^{2}\right)}\right)^{2}-D & \text { if } r<r_{0} \\
D\left(1-e^{-\alpha \frac{2}{\pi}\left(\left(r_{c u t}^{M}\right)^{2}-r_{0}^{2}\right) \tan \left(\frac{\pi}{2} \frac{r^{2}-r_{0}^{2}}{\left(r_{c u t}^{M}\right)^{2}-r_{0}^{2}}\right)}\right)^{2}-D \\
\text { if } r<r_{0}<r_{c u t}^{M} \\
0 & \text { if } r>r_{c u t}^{M}\end{cases}
\end{aligned}
$$

where $r$ is the distance between the particles, $r_{0}$ is the equilibrium distance corresponding to the minimum of energy, $D$ is the depth of the energy well, $\alpha$ is a parameter controlling the rigidity of the interaction, and $r_{c u t}^{M}$ is the cutoff distance. The potential is formulated to ensure continuity of the energy and its derivatives at the cutoff distance. Consistency between the harmonic and Morse potentials requires to consider the same equilibrium distance $r_{0}$, a depth of the energy well equal to the shift of the harmonic potential $D=V_{\text {shift }}$, and to set $\alpha=\frac{1}{r_{0}} \sqrt{\frac{K}{8 D}}$ to ensure the same rigidity of interaction. The cutoff distances $r_{c u t}^{M}$ can be chosen arbitrarily. In practice, all computations are performed with dimensionless quantities, where the unit of length is $r_{0}$ and the unit of energy $K r_{0}^{2}$, so that the only parameters that must be specified are the reduced cutoff distances $r_{\text {cut }}^{H} / r_{0}$ and $r_{c u t}^{M} / r_{0}$. Here we consider, $r_{c u t}^{H} / r_{0}=1.1$ (choice of the previous study) and $r_{c u t}^{M} / r_{0}=1.5$. The two potentials are compared in Figure 2 (top).

The second material studied is $2 \mathrm{D}$ pristine graphene. Graphene is a crystalline material made of carbon atoms arranged in a honeycomb structure (see Fig. 2) and well known for its exceptional electronic, optical, thermal and mechanical properties (Geim and Novoselov, 2007; Novoselov, 2011). For the study of failure initiation by molecular simulation, graphene is a good candidate because its high strength and moderate toughness lead to one of the smallest characteristic length $l_{c}$ (Fig. 1). Simulating graphene failure requires a potential that is able to capture the breaking and rearrangement of covalent bonds. Reactive potentials are suitable for this and various reactive potentials have been used in the literature to investigate failure properties of graphene : REBO/AIREBO (e.g., strength by Lu and Huang (2009); Zhao et al (2009), fracture by Zhang et al (2012); Dewapriya et al (2014); Han et al (2017)), Tersoff (e.g., strength by Rajasekaran et al (2016)), and ReaxFF (e.g., strength by Jensen et al (2015)). All these potentials lead to mechanical properties in reasonable agreement with experimental or ab-initio results. In the present study, we consider the second generation REBO (REactive Bond Order) potential (Brenner et al, 2002) initially proposed by Brenner (1990), which is a good compromise between accuracy and computational cost. The parameterization of REBO is known to yield an excessive strength and toughness because of a spurious bump in the aromatic carbon-carbon interaction (Shenderova et al, 2000; Belytschko et al, 2002). To solve this problem, it has been proposed to modify the function performing a smooth cutoff of the carbon-carbon interaction between $r_{\min }=1.7 \AA$ and $r_{\max }=2.0 \AA$ (Zhao et al, 2009; Lu et al, 2011; Zhang et al, 2012; Yazdani and Hatami, 2015). A simple modification consists in changing the value of $r_{\min }$ to $2.0 \AA$. With this modification, one obtains mechanical properties more consistent with experiments and ab-initio calculations (see Tab. 1 and Fig. 2 right), but the potential exhibits a discontinuity of energy at $r=2.0 \AA$. This discontinuity is of no consequence as long as none of the aromatic bonds reaches $2.0 \AA$. In all the cases studied in this work, the most stretched aromatic bonds reach $1.8 \AA$ at failure initiation. Therefore, this modification of the REBO potential is valid for the present study. In this study, we limit ourself to the study graphene in 2D. Taking into account the third dimension does affect the mechanism of failure as more favorable paths to failure can be accessed in 3D (Brochard et al, 2018). Yet, the main findings of this paper (role of stress concentration on failure mechanisms) are quite general and are expected to remain true in $3 \mathrm{D}$.

All the systems studied are periodic and mechanical loading is applied by changing the size of the periodic box. When approaching failure, the typical loading steps considered are strain increments of $\sim 10^{-5}-$ $10^{-4}$ (presence of flaws often requires smaller increments for satisfying accuracy). The evolution of the system (atomic positions) is obtained by minimizing the energy at each loading step. We investigate systems of various sizes $\left(10^{2}\right.$ to $10^{4}$ atoms) with and without flaws (see some examples in Figure 3). At each loading step, the stress (virial estimate) and the Hessian matrix are computed and the simulation is stopped when failure is observed. For the periodic systems we considered, the system fully separates in two parts at failure, which makes it easy to detect (stress drop to zero). The Hessian matrix systematically exhibits two null eigenval- 


\begin{tabular}{|c|c|c|c|c|}
\hline Method & Reference & $\begin{array}{l}\text { Young's } \\
\text { modulus } \\
(\mathrm{N} / \mathrm{m})\end{array}$ & $\begin{array}{c}\text { Uniaxial strength } \\
\qquad(\mathrm{N} / \mathrm{m}) \\
\mathrm{ZZ} / \mathrm{AC}\end{array}$ & $\begin{array}{c}\text { Mode I toughness } \\
\left(\mathrm{mN} \cdot \mathrm{m}^{-1 / 2}\right) \\
\mathrm{ZZ} \mathrm{crack} \mathrm{/} \mathrm{AC} \text { crack }\end{array}$ \\
\hline experiment & $\begin{array}{c}\text { (Lee et al, 2008) } \\
\text { (Zhang et al, 2014) }\end{array}$ & $\begin{array}{c}340 \pm 50 \\
-\end{array}$ & $\begin{array}{c}42 \pm 4 \\
-\end{array}$ & $\begin{array}{c}- \\
1.36 \pm 0.20\end{array}$ \\
\hline DFT & $\begin{array}{l}\text { (Liu et al, 2007) } \\
(\mathrm{Xu} \text { et al, 2012) }\end{array}$ & $\begin{array}{c}351 \\
-\end{array}$ & $\begin{array}{c}36.7 \text { / } 40.4 \\
-\end{array}$ & $1.40 \overline{-} / 1.24$ \\
\hline $\begin{array}{c}2^{\text {nd }} \\
\text { generation } \\
\text { REBO }\end{array}$ & $\begin{array}{c}\text { this work } \\
\text { (Arroyo and Belytschko, 2004) } \\
\text { (Lu and Huang, 2009) } \\
\text { (Zhang et al, 2012) }\end{array}$ & $\begin{array}{l}250 \\
243 \\
243 \\
-\end{array}$ & $\begin{aligned} 35.6 & / 30.5 \\
& - \\
35.6 & / 30.5 \\
& -\end{aligned}$ & $\begin{aligned} 0.77 & / 0.90 \\
& - \\
& - \\
1.02 & / 1.13\end{aligned}$ \\
\hline
\end{tabular}

Table 1 Mechanical properties of pristine graphene: experimental and ab-initio results compared to results obtained considering classical molecular modeling with the REBO potential. For REBO, we present the results of this work along with literature results using the same modification of the potential (change of the cut-off function). The difference regarding the toughness is to be attributed to the method of estimation. We adopt a method based on an analysis of the stress at failure of pre-cracked systems (Brochard et al, 2018), whereas the method of Zhang et al (2012) is based on the strain field at failure. Both methods would lead to the same results for a perfectly linear elastic material, but the significant non-linearity at the vicinity of the crack tip explains the difference.
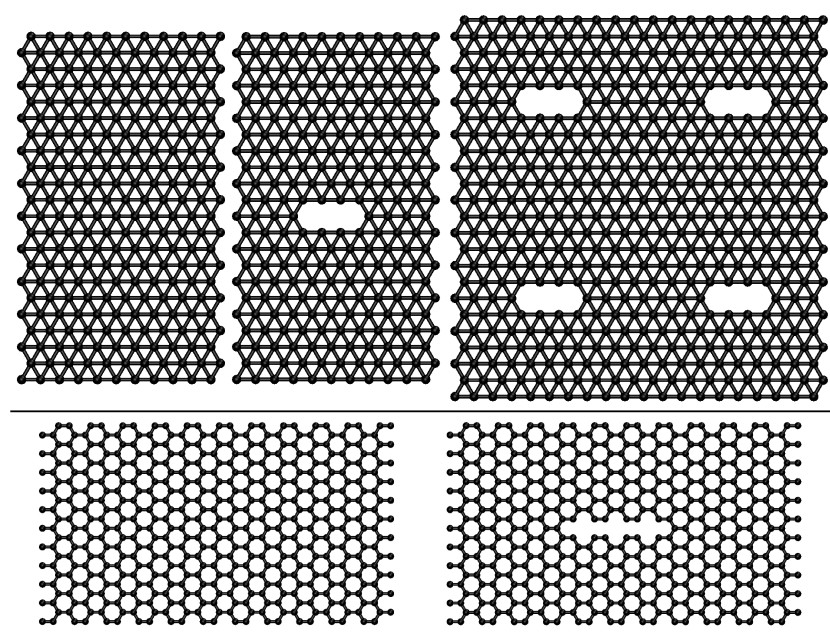

Fig. 3 Example of systems studied for the toy model (top) and for pristine graphene (bottom).

ues corresponding to the rigid body translations in $2 \mathrm{D}$, which are excluded from the analysis.

\section{Results}

We first compare the stability analysis in the athermal limit for intact and flawed materials. The results are displayed in Figure 4 for the toy model and in Figure 5 for pristine graphene. These results confirm that failure initiation corresponds to the occurrence of an instability irrespective of the stress concentration, since the smallest eigenvalue of the Hessian matrix systematically falls to zero precisely at the point of failure. The modes of failure (eigenvector) are significantly impacted by the stress concentration. In absence of stress concentration, one observes collective moves of the atoms throughout the entire system, whereas stress concentration leads to a strong localization of the mode of failure at the point of stress concentration. For the flawless system, the collective moves form two blocks or bands of homogeneous displacements in opposite directions, and the transition between those blocks/bands is a zone of deformation where failure initiates. For the flawed material, the mode of failure shows important atomic displacement only near the flaw where failure is expected. These modes of failure suggest that the mechanism of failure initiation involves a size that strongly depends on stress concentration: as large as the entire structure in absence of stress concentration, and as small as a few atoms for high stress concentrations. The concept of initiation length in Finite Fracture Mechanics (FFM) follows a similar evolution (from the size of the structure to the characteristic length). It is worth noting, however, that these modes of failure (eigenvector) do not correspond to the physical crack that emerges from the instability. For instance, in Figure 5, the flawless graphene is loaded in the zigzag direction, so that the physical crack is expected around the armchair direction. Nevertheless, the mode of failure exhibits a deformation band in the zigzag direction. Testing many different systems, it appears that the mode of failure does not corresponds to the physical cracking in general. This suggests that the description of FFM (nucleation of a crack over the initiation length) is debatable. Here, our results suggest that a failure initiation criterion should involve a concept similar to the initiation length of FFM, but which does not imply a nucleation over the same path. The eigenvector provides only the atomic movements at the onset of failure. Subsequent moves involves a cascade of rearrangements that are not predictable from the initial eigenvector.

We repeated the simulations of Figures 4 and 5 for other system sizes (see Figure 6). The modes of failure always involve blocks or bands of collective dis- 


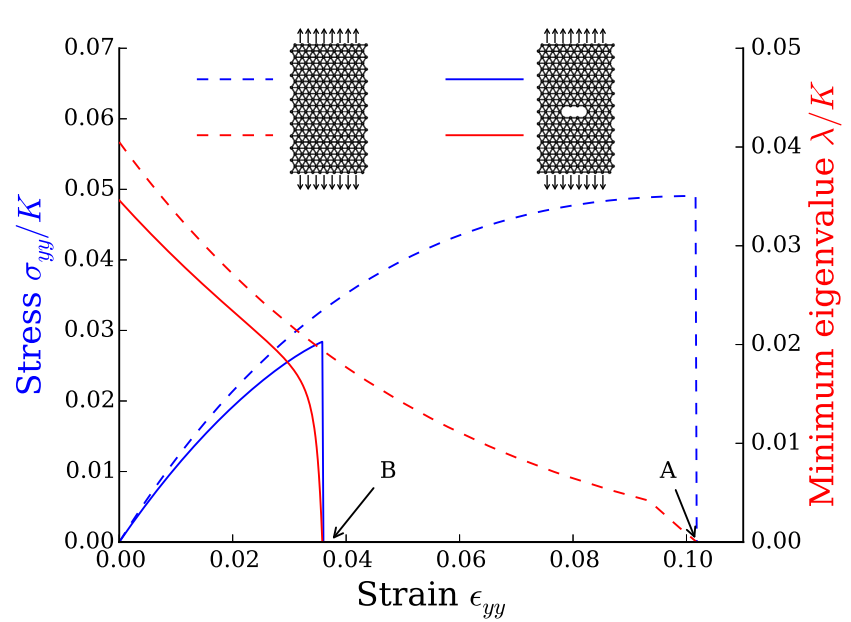

A

B
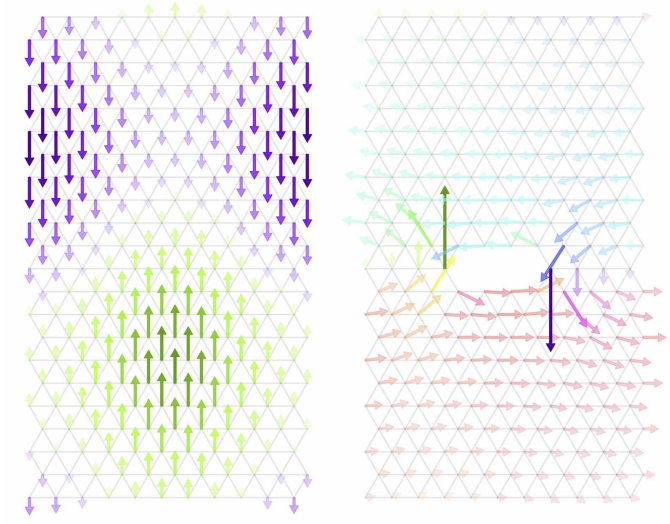

Fig. 4 Failure of the toy model with and without flaw under a loading in the $y$ direction in the athermal limit. (top) The smallest eigenvalue of the Hessian matrix falls to zero precisely when failure initiates. Failure initiation is therefore an instability at the atomic scale irrespective of stress concentration. (bottom) The associated eigenvalue provides the movements of the atoms at the onset of failure (mode of failure). The arrows provide the contribution of each atom to the eigenvector. For sake of clarity, color is used to distinguish the direction of the arrows and shading/darkness stands for the magnitude. Stress concentration strongly influences the mode of failure.

placements. Interestingly, the mode of failure of the toy model changes from blocks to bands when the dimensions of the system breaks the diagonal symmetry. The modes with blocks for the toy model exhibits the diagonal symmetry, and considering systems without this symmetry impedes the occurrence of such modes. For graphene, no impact of the diagonal symmetry could be identified, since the original mode has no diagonal symmetry. More generally, the precise nature of the mode of failure is obviously related to the boundary conditions of the system which constraints physicals symmetry. In this work, we study 2D periodic systems only, which imposes two directions of symmetry (here, vertical and horizontal). Hence, any mode of failure that spans over

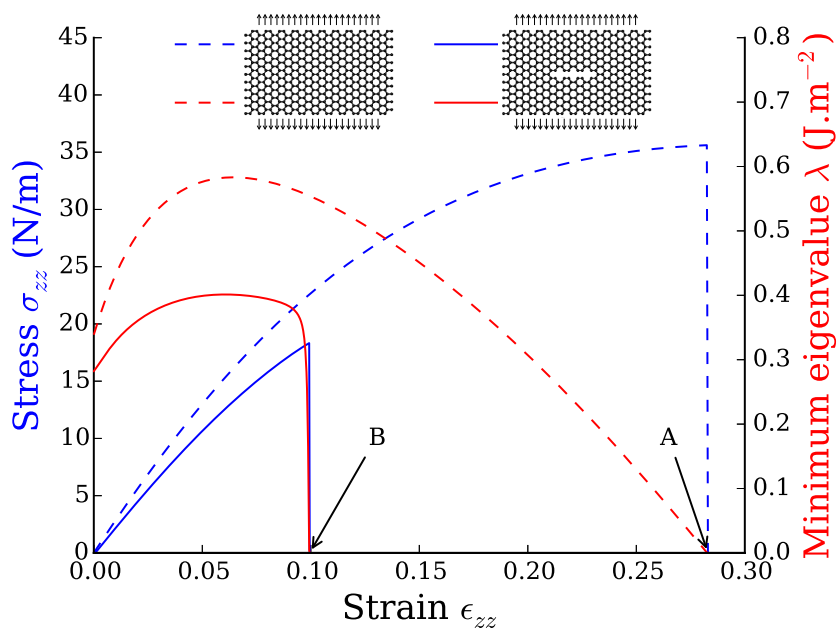

A

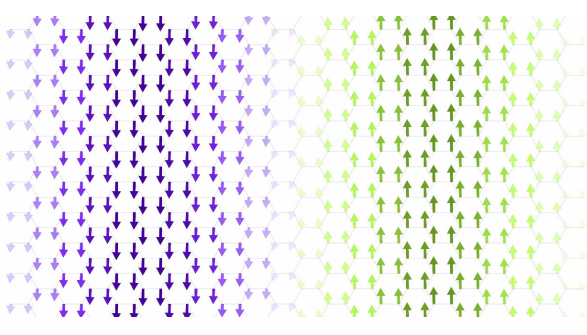

B

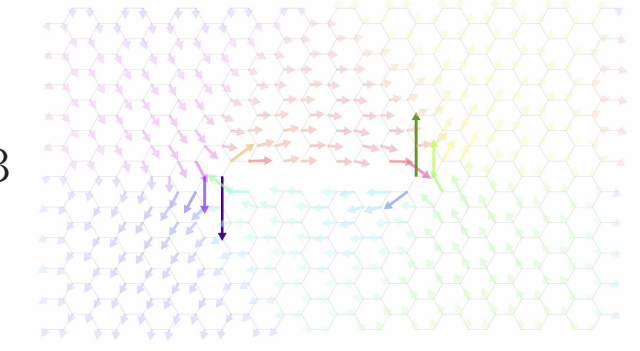

Fig. 5 Failure of pristine graphene with and without flaw under a loading in the zigzag direction in the athermal limit.

the entire structure has to respect these symmetries. In the general case, the mode of failure of flawless systems is expected to exhibit collective movements of the atoms arranged in a field that respect the specific boundary conditions of the problem (e.g., blocks/bands for periodic systems). Note, however, that the precise nature of the mode of failure has no effect on the strength of the material, i.e., on the criterion of mechanical failure. Regarding systems with flaw, one always gets localized mode of failure near the flaw, irrespective of the system size or periodicity.

Spectral analysis of the Hessian matrix points to another important effect of stress concentration (Fig. 7): flawless systems exhibit clearly two populations of modes (small and large eigenvalues separated by 2 to 3 orders of magnitudes), whereas flawed systems exhibit only the population with large eigenvalues and very few modes with small eigenvalues. This suggests a high degeneracy of the mode of failure in absence 


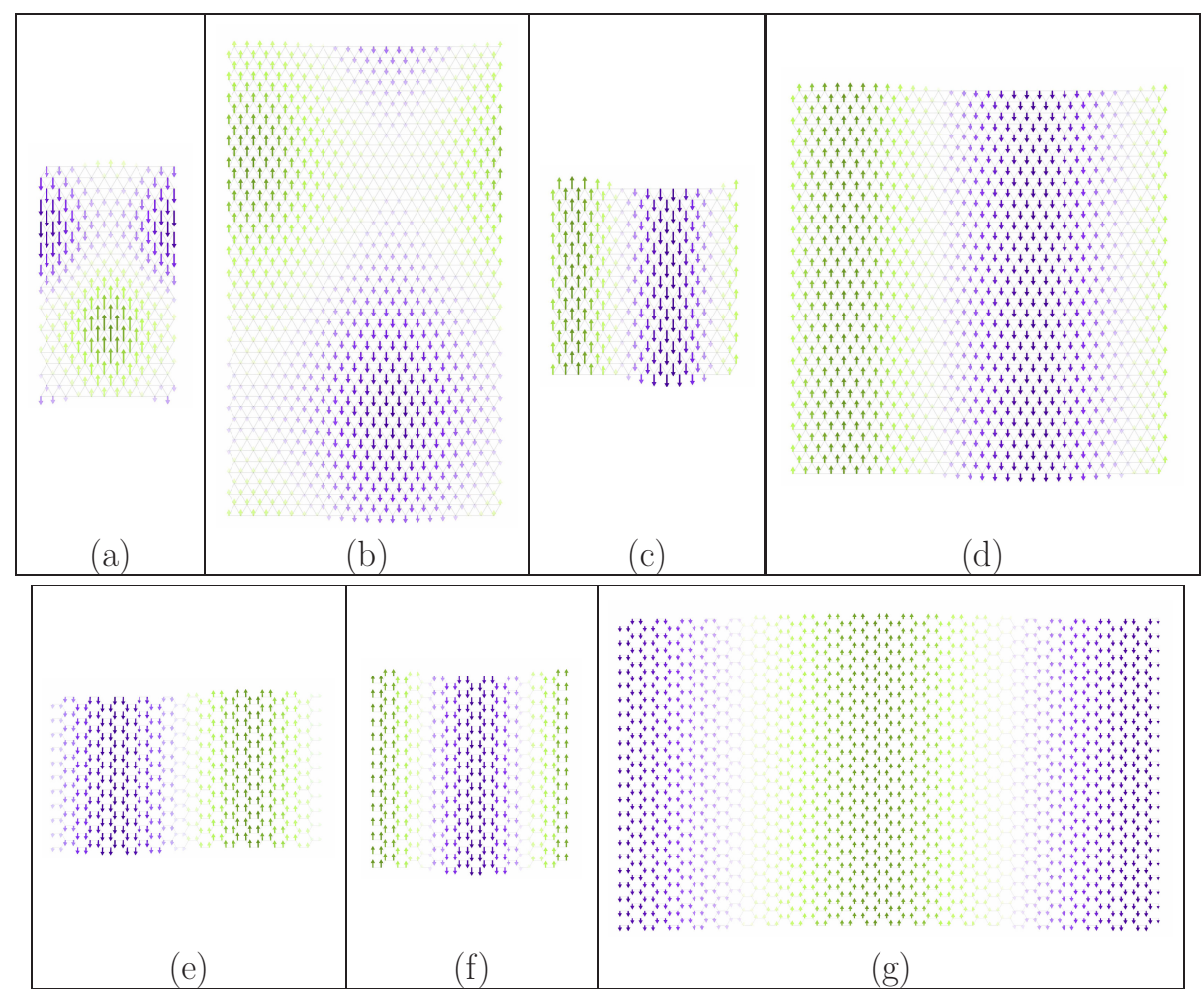

Fig. 6 Modes of failure for different system dimensions: (a) and (b) toy model with diagonal symmetry, (c) and (d) toy model without diagonal symmetry, (e) and (g) graphene with diagonal sysmetry, (f) graphene without diagonal symmetry.

of stress concentration, but little degeneracy for high stress concentrations. Note that this is not a strict degeneracy (eigenvalues are not equal). The high degeneracy in absence of stress concentration is to be attributed to the perfect physical symmetry of the mono-crystal, whereas the presence of a flaw breaks the symmetry and thus suppresses the degeneracy. Degeneracy quantifies the number of different paths to failure. At finite temperature, the number of transition paths to failure has an impact on the probability of failure. The scaling at finite temperature observed in Brochard et al (2018) for the toy model loaded in $y$ suggests that the number of transition states is proportional to the number of atoms in absence of stress concentration, and is proportional to the number of cracks under high stress concentrations.

To quantify degeneracy, we estimated the number of modes with eigenvalue below a threshold that separates the two populations (dashed line in Fig. 7 left). We display in Figure 7 (right) how this quantity scales with the system size. For flawless systems, we systematically find a linear scaling with the number of atoms. But the prefactor strongly depends on the loading direction and on the material. In contrast, for systems with flaws, we find a linear scaling with the number of flaws. This scaling is obtained by considering multiple identical periodic flaws within the same periodic box (see case with 4 flaws in Figure 3). Doing so we increase the number of transition paths to failure proportionally to the number of flaws, hence the linear scaling with the number of flaws. The scalings for the toy model are very accurate because the two populations of modes are well separated. In particular we find precisely one mode per flaw. The case of graphene is more questionable because the two populations are not so well separated. We find about 12 modes per flaw, but this seems a lot, since one would expected only very few transition paths to failure for each flaw. Anyway, stress concentration dramatically decreases the degeneracy of the mode of failure to a few modes per flaw.

To investigate the case of intermediate stress concentrations, we consider the three flaws of Figure 8: a crack (maximum stress concentration), a half-notch (intermediate) and a notch (smallest). In this work, it is not possible to consider any arbitrary stress concentration, because a flaw must comply with the crystallography of the mono-crystal. In Figure 8, we display the loading curves (stress and minimum eigenvalue), mode of failure, and statistical distribution of eigenvalues for the three systems. The differences between the three situations are not very pronounced. Yet, one can make the following observations: stress concentration enhances the variations of the minimum eigenvalue near failure, and favors higher eigenvalues in the statistical 

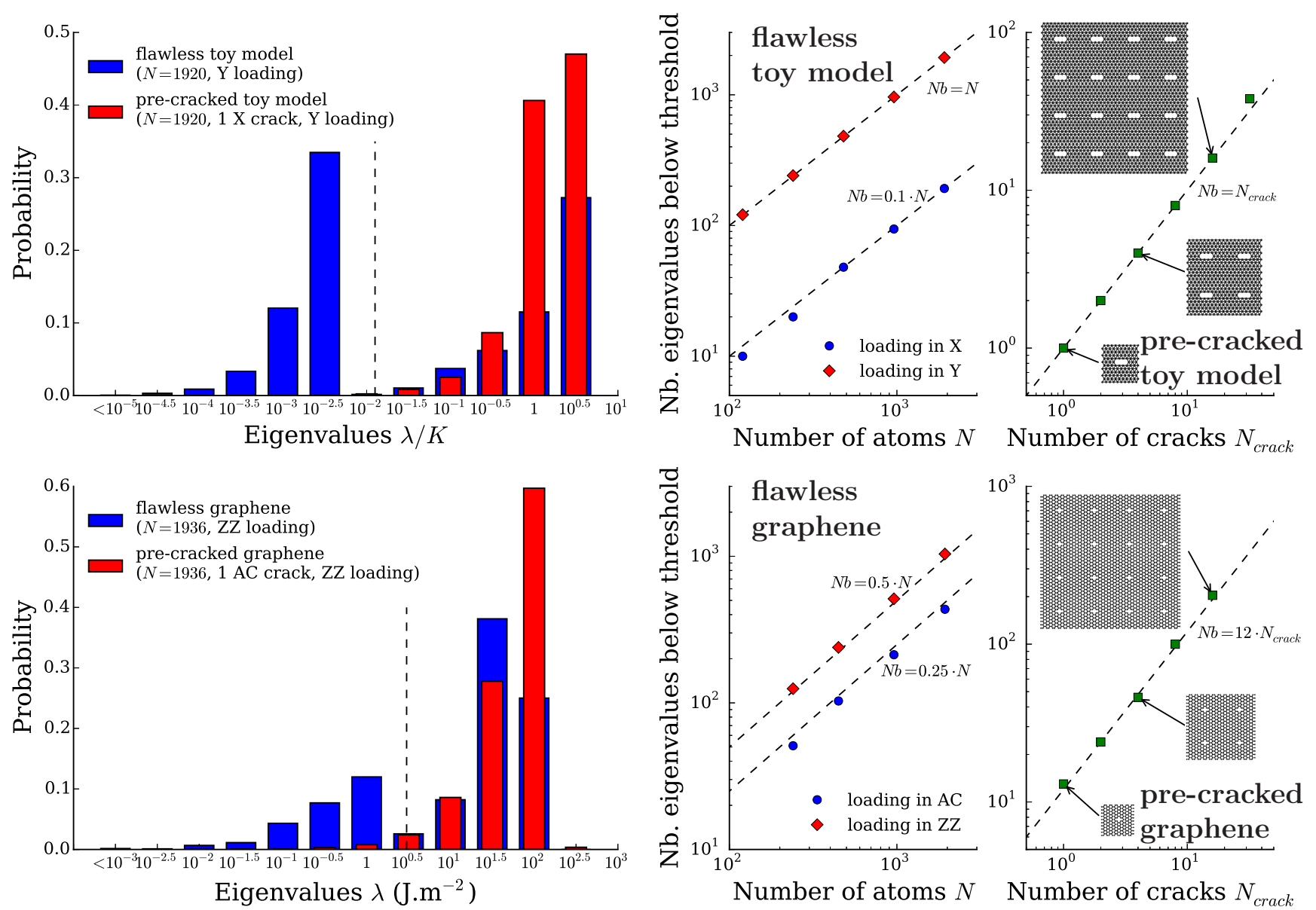

Fig. 7 Statistical distribution of the eigenvalues of the Hessian matrix at mechanical failure for the toy model (top left) and for pristine graphene (bottom left) with and without flaw. Two population are identified separated by order of magnitude. The number of modes with small eigenvalues is interpreted as a degeneracy of the mode of failure. It is quantified by evaluating the number of modes below a threshold (dashed line) separating the two populations. This quantity is found to scale linearly with the number of atoms for flawless systems and with the number of cracks for systems with flaws (right plots).

distribution. The modes of failure are all very localized and the degeneracy of the mode of failure is unaffected (number of eigenvalues below $10^{-2}$ ). For the crack and half-notch cases, the loading curves near failure exhibit a softening branch (see small charts in Figure 8). We verified that this softening is not the onset of failure by performing an unloading (dotted lines) which proves that the behavior is perfectly reversible. This softening is due to the most stretched bonds at the tip which has entered the softening branch of the bond force (see Fig. 2) (the most stretched bonds are highlighted in black in Figure 8). The failure occurs after some softening precisely when the smallest eigenvalue of the Hessian reaches 0 . The notch and half notch configurations are typically cases where macroscopic theory faces contradictions between stress and energy criteria. Here, we confirm that instability does capture failure initiation, even when peculiar mechanical non linearities arise such as the softening discussed above.

\section{Discussion}

The results of this study identify instability as the elementary mechanism of brittle failure in the athermal limit, irrespective of stress concentration. But stress concentration strongly impact the mode of instability (localized or not) and its degeneracy (highly degenerated or not). The mode of instability provides the direction of atomic moves at the onset of failure, but subsequent evolution of the system is inherently out of equilibrium. The system can bifurcate into a wide variety of configurations and does not follow the path of minimum energy in general. Thus, actual cracking cannot be predicted from the mode of instability. For instance, the actual cracking of graphene is displayed in Figure 9 ). It is obtained by low temperature molecular dynamics as in Brochard et al (2018). Significant kinetic energy is released as failure proceeds, which dominates the dynamics of cracking since this kinetic energy much exceeds that provided by the thermostat. 

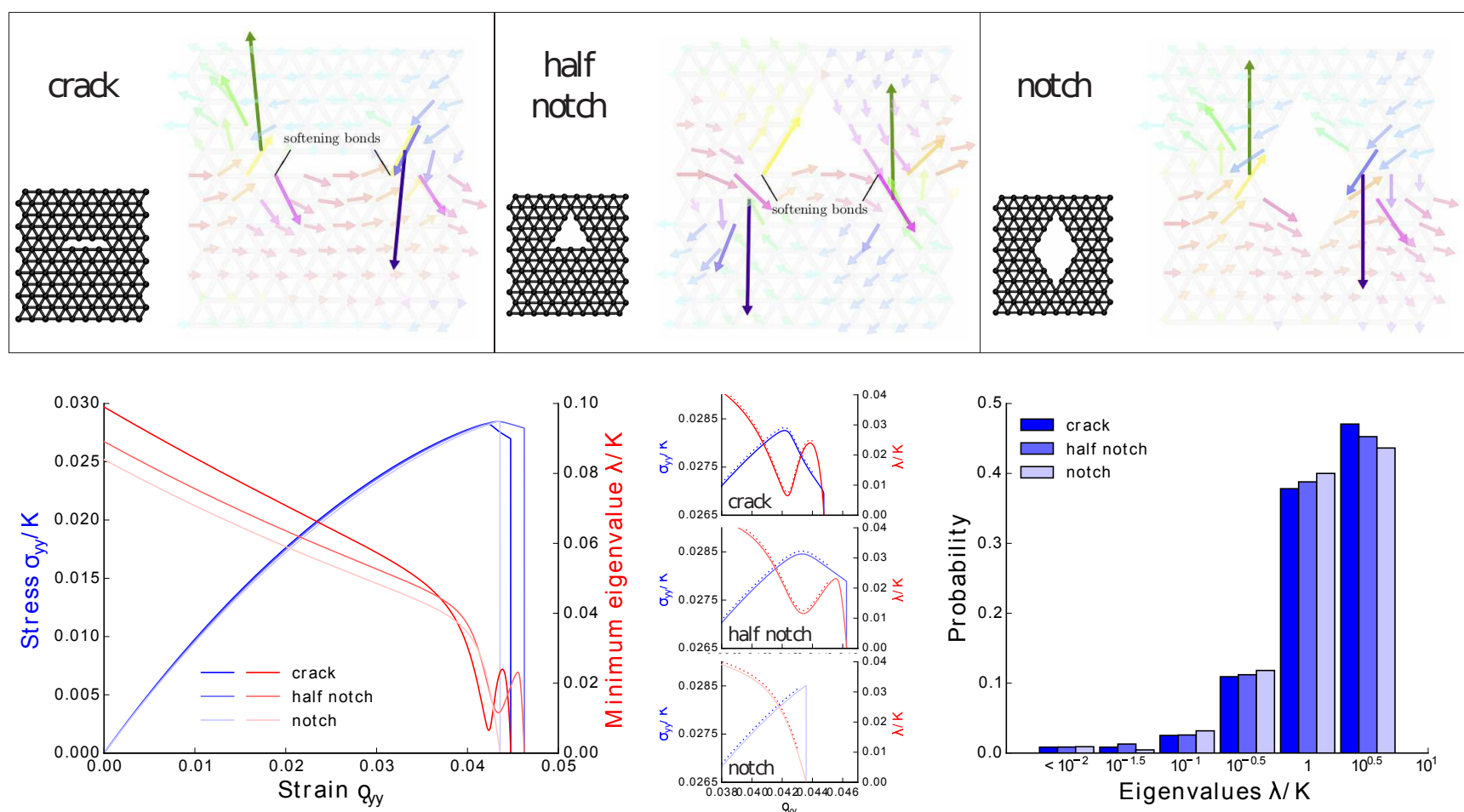

Fig. 8 Loading curves (stress and minimum eigenvalue), modes of failure and statistical distribution of modes for the toy model with three different flaws of varying stress concentration: crack, half notch, and notch (in order of decreasing stress concentration). The small charts are zooms of the loading curves near failure. In these charts, the dotted lines are an example of unloading. These dotted lines prove that the behavior is fully reversible even in the softening branches (the dotted lines are slightly shifted vertically for proper visualization). The onset of irreversibility and failure is precisely at the point of instability. The softening branches are attributed to the most stretched bonds at the tips identified in black in the modes of failure of the crack and half notch cases.

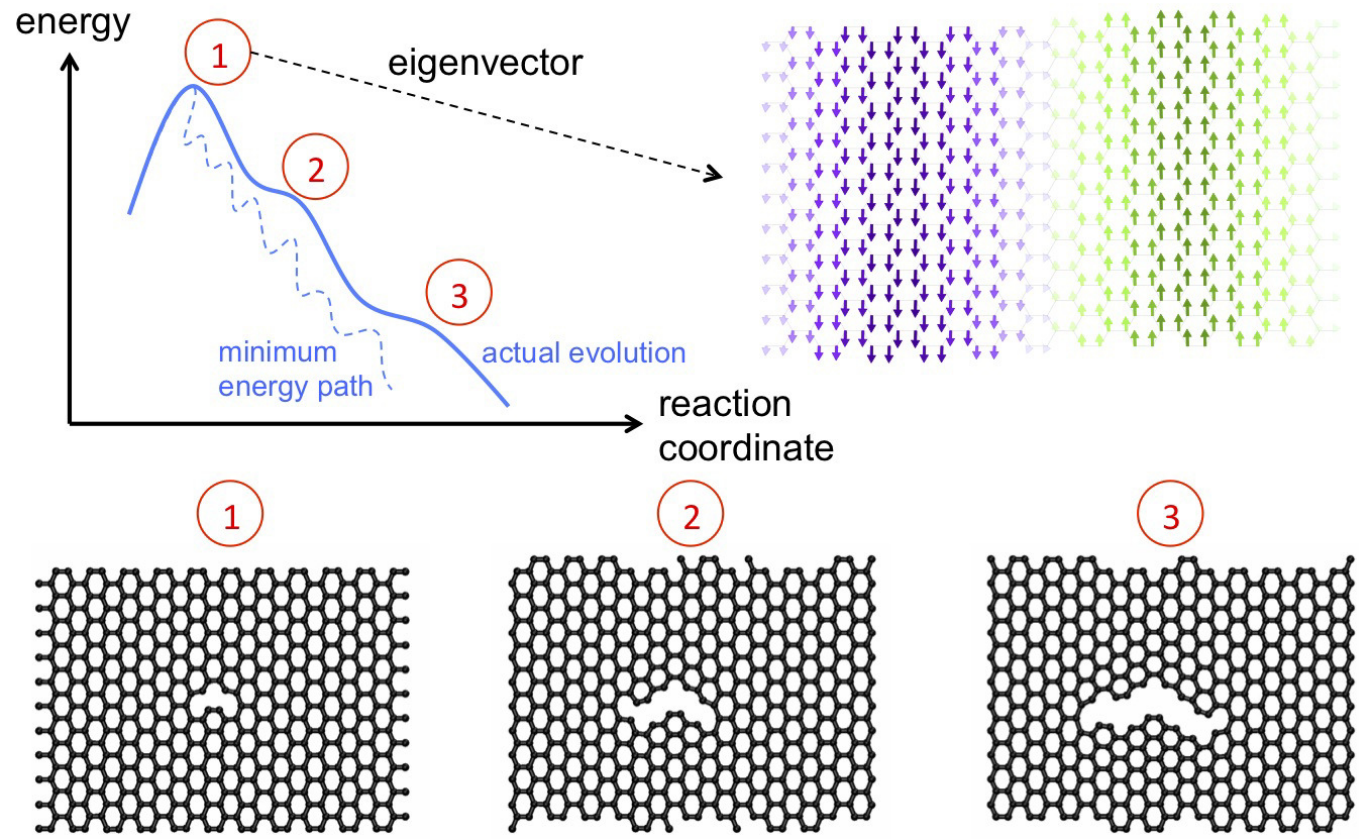

Fig. 9 Actual cracking does not follow the mode of instability at initiation. After the transition state is reached, the evolution of the system follows a dynamics out of equilibrium, and not necessarily the path of minimum energy. 
With respect to the macroscopic description of failure initiation, this study suggests that an appropriate description should refer to instability as a general criterion for failure. And the difference between energyand stress-driven failures originates from the degree of localization of the associated mode of instability:

- In absence of stress concentration, the strain and stress fields are homogeneous and the mode of instability is collective. Therefore, the instability corresponds to an instability with respect to the homogeneous strain tensor. It corresponds to a singular stiffness tensor $(\operatorname{det}(\mathbb{C})=0)$, and since the stiffness tensor is a function of stress, this is a stress-driven failure.

- Under high stress concentration, the strain and stress fields exhibit a singularity at the point of concentration. Yet, singular fields are an idealization of reality and there exists a minimum length scale below which this idealization collapses. The characteristic length $l_{c}=\left(K_{c} / \sigma_{c}\right)^{2}$ is an estimate of this minimum scale (4-5 atoms for graphene). $l_{c}$ is the scale of localization of the mode of instability at failure initiation. Accordingly, from a continuum perspective, instability under high stress concentration is characterized by a singular stiffness tensor over a volume of length scale $l_{c}$. Instability is therefore analogous to a stress criterion over a length $l_{c}$, which is the basis of the non local theory (Novozhilov, 1969). Because of the $r^{-1 / 2}$ stress singularity at crack tips, a stress criterion over a length $l_{c}$ is equivalent to a criterion on toughness, and therefore, we recover an energydriven failure .

A criterion based on instability only provides the onset of failure. A small crack nucleus appears (not the size of the mode of instability), and subsequent evolution follows a propagation dynamics distinct from the mode of instability. Accordingly, one should clearly distinguish between the onset of failure (failure initiation) and the cracking (failure propagation). In this work, we find that failure initiation is captured by instability for a perfectly brittle material, i.e., with no irreversibilities prior to failure. Irreversibilities such as damage or plasticity introduce additional instabilities (e.g., microcrack nucleations, dislocation formations and moves), and therefore macroscopic failure initiation is no more associated to the first instability. Instead, a series of instabilities (cascade of irreversibilities) is expected to occur before the macroscopic failure.

Another important finding is that stress concentration impacts the degeneracy of the mode of failure. This is of importance at finite temperature, since degeneracy quantifies the number of transition states to failure.
The mode of failure is highly degenerated in absence of stress concentration, but very little degenerated in presence of a crack. Of course, degeneracy is expected to be closely related to the physical symmetries of the system, which is broken by the presence of a flaw. As soon as a little stress concentration is introduced, degeneracy is immediately removed and the transition paths to failure exhibit different energies. Only the path of smallest energy is reached in the athermal limit, but at finite temperature, competition between the different paths is significant if the differences between transition path energies are of the order of the thermal agitation energy. As stress concentration is increased, the difference of energies are exacerbated and the system is expected to fail via the transition path of minimum energy only.

In a previous work on failure at finite temperature (Brochard et al, 2018), we show that strength and toughness of mono-crystals follow a universal scaling law in temperature, loading rate and system size:

$$
\frac{K_{c}}{K_{c}(0 K)} \text { or } \frac{\sigma_{c}}{\sigma_{c}(0 K)}=f\left(\frac{T}{T_{0}} \ln \left(\frac{N_{T S}}{\dot{\epsilon} / \dot{\epsilon}_{0}}\right)\right)
$$

where $T_{0}$ and $\dot{\epsilon}_{0}$ are constants and $f$ is a function that depends on the material. For pristine graphene, $f(x)=$ $1-x$, which is the well-known Zhurkov's law, predicting the strength of many brittle materials at low and moderate temperatures (Zhurkov, 1984). Zhurkov's law disregards size effects, but it appears that the scalings of strength and toughness differ precisely on this aspect. The dependence to system size is accounted for through the quantity $N_{T S}$ which refers to the number of transition states. In Brochard et al (2018), by analyzing the scaling for the toy model loaded in $y$, we infer that $N_{T S}$ is proportional to the number of atoms for strength, and $N_{T S}$ is proportional to the number of cracks for the toughness. The number of transition states $N_{T S}$ is expected to relate directly to the degeneracy in the athermal limit. The scaling of degeneracy in Fig. 7 (right) proves that the scaling with size we infer in our previous study is correct. Investigating degeneracy shows that the prefactors of these scalings depend on the material and loading direction. But the exponent is systematically 1 (linear), which confirms the general scaling found at finite temperature. It is interesting to note that, with this determination of $N_{T S}$, all the constants in the scaling law (3), expect the characteristic time scale $\left(1 / \dot{\epsilon}_{0}\right)$, can be established from the athermal limit. The values of $T_{0}$ and the expression of $f$ can be obtained by estimating the energy barrier to transition states (see the case of pristine graphene in Brochard et al (2018)).

Finally, one may wonder whether existing continuum theories of brittle failure initiation are consistent 
with our atomistic description. A first issue is that most existing theories do not clearly distinguish the initiation mechanism from the physical cracking. Here we find that initiation follows a mode of instability that is related to the physical cracking only through the crack nucleus that form at the onset of failure. Phase Field (PF), Cohezive Zone Model (CZM), and Finite Fracture Mechanics (FFM) theories do elaborate the failure criterion on the physical crack that is expected to form. Non Local (NL) theory is the only theory in which the initiation mechanism may be distinct from the subsequent cracking. An other important aspect is that the volume involved in failure initiation varies widely depending on stress concentration. PF, CZM and FFM theories also involve different volumes depending on stress concentration, but this is not the case of NL theory. Accordingly, none of the existing theories is fully consistent with the atomistic description. A requirement that guided the formulation of existing continuum theories, is the introduction of the characteristic length $l_{c}=\left(K_{c} / \sigma_{c}\right)^{2}$. Indeed, usual continuum mechanics based on stress and strains does not incorporate any internal length, so it has to be complemented to capture at the same time stress driven failure and energy driven failure. Our results suggest that the requirement should be to capture atomic instabilities and not just introducing an internal length. Higher order gradient theories have been used successfully to capture instabilities and strain localization for systems without stress concentration (Triantafyllidis and Bardenhagen, 1993; Bardenhagen and Triantafyllidis, 1994). The challenge is to find an enriched formulation that would capture instabilities for any stress concentration. This is an objective for future research.

\section{Conclusion}

In this study, we investigated the elementary mechanisms of brittle failure initiation in mono-crystals by molecular simulation in the athermal limit. We find that:

- Failure initiates as an instability, irrespective of the stress concentration, which suggests that a universal macroscopic criterion should be based on instability analysis.

- Stress concentration has a strong impact on the mode of failure, in particular its spatial extent (very localized for high stress concentration, distributed throughout the system in absence of stress concentration), and its degeneracy (degeneracy related to the number of flaws for high stress concentrations, and related to the number of atoms in absence of stress concentration).

- The degeneracy of the mode of failure quantifies the number of different paths to failure, which can be related to the scaling of strength and toughness at finite temperature. In particular, it explains why strength of mono-crystals scales with the number of atoms, whereas toughness scales with the number of cracks.

- None of the existing theories of brittle failure initiation is fully consistent with the atomistic description (initiation not related to subsequent cracking and volume involved varies with stress concentration). Existing formulations primarily aim at introducing the characteristic length $l_{c}=\left(K_{c} / \sigma_{c}\right)^{2}$ in the continuum mechanics. A fully consistent theory should rather aim at capturing the atomic instabilities. This has been done successfully in absence of stress concentration (e.g., higher order gradient theories), but a theory adapted to any stress concentration is still to be proposed.

Acknowledgements The authors are grateful to E. Cances, F. Legoll, T. Lelivre, and G. Stoltz for stimulating discussions and constant encouragements. We also gratefully acknowledge funding from the Labex MMCD provided by the national program Investments for the Future of the French National Research Agency (ANR-11-LABX-022-01)

\section{References}

Arroyo M, Belytschko T (2004) Finite crystal elasticity of carbon nanotubes based on the exponential Cauchy-Born rule. Physical Review B 69(11):115,415, DOI 10.1103/PhysRevB.69.115415, URL https://link.aps.org/doi/10.1103/PhysRevB.69.115415

Ashby MF (2005) Materials Selection in Mechanical Design, 3rd edn. Butterworth-Heinemann (Elsevier, Inc.)

Bardenhagen S, Triantafyllidis N (1994) Derivation of higher order gradient continuum theories in 2,3-d non-linear elasticity from periodic lattice models. Journal of the Mechanics and Physics of Solids 42(1):111139, DOI 10.1016/0022-5096(94)90051-5, URL http://linkinghub.elsevier.com/retrieve/pii/0022509694900515

Belytschko T, Xiao SP, Schatz GC, Ruoff RS (2002) Atomistic simulations of nanotube fracture. Physical Review B 65(23):235,430, DOI 10.1103/PhysRevB.65.235430, URL http://link.aps.org/doi/10.1103/PhysRevB.65.235430 
Brenner DW (1990) Empirical potential for hydrocarbons for use in simulating the chemical vapor deposition of diamond films. Physical Review B 42(15):9458-9471, DOI 10.1103/PhysRevB.46.1948.2, URL http://link.aps.org/doi/10.1103/PhysRevB.42.9458

Brenner DW, Shenderova OA, Harrison JA, Stuart SJ, Ni B, Sinnott SB (2002) A second-generation reactive empirical bond order (REBO) potential energy expression for hydrocarbons. Journal of Physics: Condensed Matter 14(4):783-802, DOI 10.1088/09538984/14/4/312, URL http://stacks.iop.org/0953$8984 / 14 / \mathrm{i}=4 / \mathrm{a}=312$ ?key $=$ crossref.563ba867ec801ce55

Brochard L, Tejada IG, Sab K (2016) From yield to fracture, failure initiation captured by molecular simulation. Journal of the Mechanics and Physics of Solids 95:632646, DOI 10.1016/j.jmps.2016.05.005, URL

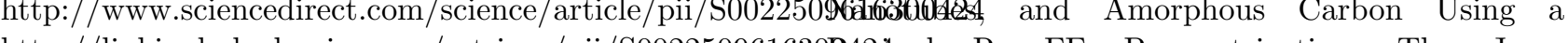
http://linkinghub.elsevier.com/retrieve/pii/S002250961630B\&\&ilsed ReaxFF Parametrization. The Jour-

Brochard L, Souguir S, Sab K (2018) Scaling of brittle failure: strength versus toughness. International Journal of Fracture 210(1-2):153166, DOI 10.1007/s10704-018-0268-9, URL http://link.springer.com/10.1007/s10704-018-0268-9

Dailidonis V, Ilyin V, Mishra P, Procaccia I (2015) Consequences of disorder on the stability of amorphous solids. Physical Review B 92(9):094,105, DOI 10.1103/PhysRevB.92.094105, URL https://link.aps.org/doi/10.1103/PhysRevB.92.094105, Lee 1507.01207

Delph T, Zimmerman J, Rickman J, Kunz J (2009) A local instability criterion for solid-state defects. Journal of the Mechanics and Physics of Solids 57(1):67-75, DOI 10.1016/j.jmps.2008.10.005, URL https://linkinghub.elsevier.com/retrieve/pii/S00225096

Dewapriya MAN, Rajapakse RKND, Phani AS (2014) Atomistic and continuum modelling of temperature-dependent fracture of graphene. International Journal of Fracture 187(2):199212, DOI 10.1007/s10704-014-9931-y, URL http://link.springer.com/10.1007/s10704-014-9931-y

Dugdale D (1960) Yielding of steel sheets containing slits. Journal of the Mechanics and Physics of Solids 8(2):100-104, DOI 10.1016/0022-5096(60)90013-2

Elliott RS, Shaw JA, Triantafyllidis N (2006) Stability of crystalline solidsII: Application to temperature-induced martensitic phase transformations in a bi-atomic crystal. Journal of the Mechanics and Physics of Solids 54(1):193232, DOI 10.1016/j.jmps.2005.07.008, URL https://linkinghub.elsevier.com/retrieve/pii/S00225096050
Geim AK, Novoselov KS (2007) The rise of graphene. Nature Materials 6(3):183191, DOI 10.1038/nmat1849, URL http://www.nature.com/articles/nmat1849, 0702595

Han J, Sohn D, Woo W, Kim DK (2017) Molecular dynamics study of fracture toughness and trans-intergranular transition in bi-crystalline graphene. Computational Materials Science 129:323331, DOI 10.1016/j.commatsci.2016.12.023, URL http://dx.doi.org/10.1016/j.commatsci.2016.12.023 https://linkinghub.elsevier.com/retrieve/pii/S0927025616306462

Hsieh C, Thomson R (1973) Lattice theory of crack creep. Journal of Applied Physics 44(5):2051-2063, DOI 10.1063/1.1662512, URL http://aip.scitation.org/doi/10.1063/1.1662512

Jensen BD, Wise KE, Odegard GM (2015) Simulation of the Elastic and Ultimate Tensile nal of Physical Chemistry A 119(37):97109721, DOI 10.1021/acs.jpca.5b05889, URL http://pubs.acs.org/doi/10.1021/acs.jpca.5b05889

Kitamura T, Umeno Y, Tsuji N (2004) Analytical evaluation of unstable deformation criterion of atomic structure and its application to nanostructure. Computational Materials Science 29(4):499510, DOI 10.1016/j.commatsci.2003.12.004, URL https://linkinghub.elsevier.com/retrieve/pii/S0927025604000059 ee C, Wei X, Kysar JW, Hone J (2008) Measurement of the Elastic Properties and Intrinsic Strength of Monolayer Graphene. Science 321(5887):385388, DOI 10.1126/science.1157996, URL http://www.ncbi.nlm.nih.gov/pubmed/18635798 http://www.sciencemag.org/cgi/doi/10.1126/science.1157996, $6080 @ 7749150628$

Leguillon D (2002) Strength or toughness? A criterion for crack onset at a notch. European Journal of Mechanics, A/Solids 21(1):61-72, DOI 10.1016/S09977538(01)01184-6

Li X (2013) A bifurcation study of crack initiation and kinking. The European Physical Journal B 86(6):258, DOI 10.1140/epjb/e2013-40145-9, URL http://link.springer.com/10.1140/epjb/e201340145-9

Liu F, Ming P, Li J (2007) Ab initio calculation of ideal strength and phonon instability of graphene under tension. Physical Review B 76(6):064,120, DOI 10.1103/PhysRevB.76.064120, URL http://link.aps.org/doi/10.1103/PhysRevB.76.064120 Lu Q, Huang R (2009) Nonlinear mechanics of 0.8ingtXatomic-layer graphene sheets. International Journal of Applied Mechanics 01(03):443- 
467, DOI 10.1142/S1758825109000228, URL Rajasekaran G, Kumar R, Parashar A (2016) http://www.worldscientific.com/doi/abs/10.1142/S175882511960102\$8tential with improved accuracy for

$\mathrm{Lu}$ Q, Gao W, Huang R (2011) Atomistic simulation and continuum modeling of graphene nanoribbons under uniaxial tension. Modelling and Simulation in Materials Science and Engineering 19(5):054,006, DOI 10.1088/09650393/19/5/054006, URL http://stacks.iop.org/09650393/19/i=5/a =054006?key=crossref.f60aadba1ef1fc

Maloney CE, Lemaître A (2006) Amorphous systems in athermal, quasistatic shear. Physical Review E 74(1):016,118, DOI 10.1103/PhysRevE.74.016118, URL https://link.aps.org/doi/10.1103/PhysRevE.74.016118, 0510677

Marder M, Gross S (1994) Origin of Crack Tip Instabilities. Journal of the Mechanics and Physics of Solids 43(1):1-48, DOI 10.1016/0022-5096(94)00060-I, URL http://arxiv.org/abs/chao-dyn/9410009, 9410009

Miehe C, Welschinger F, Hofacker M (2010) Thermodynamically consistent phase-field models of fracture: Variational principles and multifield $\mathrm{FE}$ implementations. International Journal for Numerical Methods in Engineering 83(10):1273-1311, DOI 10.1002/nme.2861, URL http://doi.wiley.com/10.1002/nme.2861

Milstein F, Hill R (1979) Theoretical properties of cubic crystals at arbitrary pressureIII. Stability. Journal of the Mechanics and Physics of Solids 27(3):255279, DOI 10.1016/0022-5096(79)90004-8, URL http://linkinghub.elsevier.com/retrieve/pii/00225096

Novoselov KS (2011) Nobel Lecture: Graphene: Materials in the Flatland. Reviews of Modern Physics 83(3):837-849, DOI 10.1103/RevModPhys.83.837, URL https://link.aps.org/doi/10.1103/RevModPhys.83.837

Novozhilov V (1969) On a necessary and sufficient criterion for brittle strength. Journal of Applied Mathematics and Mechanics 33(2):201210, DOI 10.1016/0021-8928(69)90025-2, URL http://linkinghub.elsevier.com/retrieve/pii/00218928

Plimpton S (1995) Fast Parallel Algorithms for Short-Range Molecular Dynamics. Journal of Computational Physics 117(1):119, DOI 10.1006/jcph.1995.1039, URL http://linkinghub.elsevier.com/retrieve/pii/S002199918

Pratapa PP, Suryanarayana P (2016) On numerically predicting the onset and mode of instability in atomistic systems. Mechanics Research Communications 78:27-33, DOI $\quad 10.1016 /$ j.mechrescom.2016.05.001, URL http://dx.doi.org/10.1016/j.mechrescom.2016.05.001 https://linkinghub.elsevier.com/retrieve/pii/S0093641316310046iX/aip.scitation.org/doi/10.1063/1.4754115 simulating graphene in molecular dynamics environment. Materials Research Express 3(3):035,011, DOI 10.1088/2053-1591/3/3/035011, URL 1591/3/3/035011 http://stacks.iop.org/2053$1591 / 3 / \mathrm{i}=3 / \mathrm{a}=035011$ ?key $=$ crossref.d71622bf411b1381210d856e0

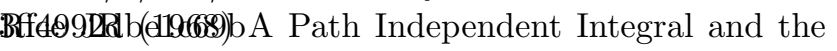
Approximate Analysis of Strain Concentration by Notches and Cracks. Journal of Applied Mechanics 35(2):379, DOI 10.1115/1.3601206

Shenderova Oa, Brenner DW, Omeltchenko a, Su X, Yang LH (2000) Atomistic modeling of the fracture of polycrystalline diamond. Physical Review B 61(6):38773888, DOI 10.1103/PhysRevB.61.3877, URL http://link.aps.org/doi/10.1103/PhysRevB.61.3877\nhttp://prb.a

Thomson R (1986) Physics of Fracture. In: Ehrenreich H, Turnbull D (eds) Solid state physics, vol 39, Academic Press, pp 1-129, DOI 10.1016/S0081-1947(08)60368-9, URL http://linkinghub.elsevier.com/retrieve/pii/S0081194708603689

Thomson R, Hsieh C, Rana V (1971) Lattice trapping of fracture cracks. Journal of Applied Physics 42(8):3154-3160, DOI 10.1063/1.1660699

Triantafyllidis N, Bardenhagen S (1993) On higher order gradient continuum theories in 1-D nonlinear elasticity. Derivation from and comparison to the corresponding discrete models. Journal of Elastic01833(3):259-293, DOI 10.1007/BF00043251, URL http://link.springer.com/10.1007/BF00043251

Williams M (1952) Stress Singularities Resulting From Various Boundary Conditions in Angular Corners of Plates in Extension. Journal of Applied Mechanics 19(4):526-528

Xu M, Tabarraei A, Paci JT, Oswald J, Belytschko T (2012) A coupled quantum/continuum mechanics study of graphene fracture. International Journal of Fracture 173(2):163900253, DOI 10.1007/s10704-011-9675-x, URL http://link.springer.com/10.1007/s10704-011-9675-x Yazdani H, Hatami K (2015) Failure criterion for graphene in biaxial loadinga molecular dynamics study. Modelling and Simulation in Materials Science 71:030XEngineering 23(6):065,004, DOI 10.1088/09650393/23/6/065004, URL http://stacks.iop.org/0965$0393 / 23 / \mathrm{i}=6 / \mathrm{a}=065004$ ?key $=$ crossref. $66817 \mathrm{f} 771850338 \mathrm{a} 194 \mathrm{c} 2 \mathrm{~b} 825$

Zhang B, Mei L, Xiao H (2012) Nanofracture in graphene under complex mechanical stresses. Applied Physics Letters 101(12):121,915, DOI $10.1063 / 1.4754115$, URL 
Zhang P, Ma L, Fan F, Zeng Z, Peng C, Loya PE, Liu Z, Gong Y, Zhang J, Zhang X, Ajayan PM, Zhu T, Lou J (2014) Fracture toughness of graphene. Nature Communications 5(1):3782, DOI 10.1038/ncomms4782, URL http://dx.doi.org/10.1038/ncomms4782 http://www.nature.com/articles/ncomms4782

Zhao H, Min K, Aluru NR (2009) Size and chirality dependent elastic properties of graphene nanoribbons under uniaxial tension. Nano Letters 9(8):3012-3015, DOI 10.1021/nl901448z, 68949092055

Zhurkov SN (1984) Kinetic concept of the strength of solids. International Journal of Fracture 26(4):295-307, DOI 10.1007/BF00962961, URL http://link.springer.com/10.1007/BF00962961 